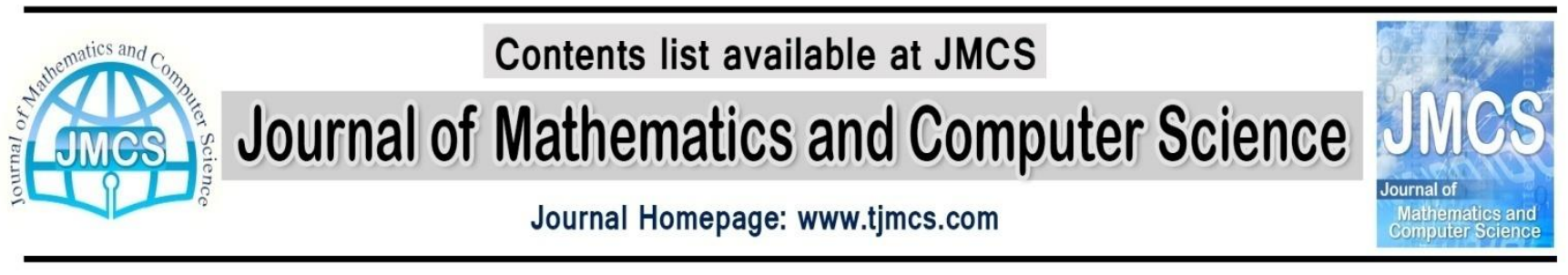

\title{
Control of Puma-560 robot Using Feedback Linearization control method and kalman filter estimator for Regulation and Tracking Purpose
}

\author{
Ehsan Zakeri ${ }^{1 *}$, Seyed Alireza Moezi ${ }^{1}$, Mehdi Zare ${ }^{2}$, Mostafa Parnian Rad $^{2}$ \\ ${ }^{1}$ Department of Mechanical Engineering, Shiraz branch, Islamic Azad University, Shiraz, Iran. \\ ${ }^{2}$ Department of Mechanical Engineering, University of Sistan and Baluchestan, Zahedan, Iran. \\ "(Corresponding author) Tel: +98-711-8370651, Email: ehsan8631@gmail.com
}

Article history:

Received May 2014

Accepted June 2014

Available online July 2014

\begin{abstract}
This research is presented to control a PUMA 560 robot which is well-known industrial robot with six degrees of freedom. It is a RRRRRR robot type which can do various tasks such as point welding in automotive industry and similar industries. The mathematical model is derived from dynamical equations by the means of Euler-Lagrange method. Stochastic feedback linearization with Kalman filter controller is implemented to control the PUMA 560 robot end effector.The regulation and tracking results are represented. The controller is examined in normal situation and even in presence of disturbances. Finally, the results indicate good performance of the controller.
\end{abstract}

Keywords: Feedback linearization, Kalman filter, PUMA 560, Nonlinear system.

\section{Introduction}

The greater usage of robot have progressed in industrial system in compare to human. It has very clear reasons. For instance, unlike the human, robots are able to work tirelessly and do repetitive tasks with high speed and accuracy. These are miniature reasons which are led to widespread use of robotic systems in various industries. One of the important challenge for robot arm is the position of the robot end effector [1]. Effective results of the robotic system usage depend on robot control and error minimization discussions. PUMA 560 is one of the most common robots in industries. This robot has six degrees of freedomthat three of them are related to manipulator and the remaining are related to end effector.Because of nonlinear nature of robot, many different nonlinear controllershave been proposed to control the robot.The nonlinear feedback control for PUMA 560is reported by Bejczy et al [2]. Fuzzy logic controller is an effective method to control the nonlinear system. in [3] fuzzy theory set and fuzzy controller are usedto estimate transference rate of positive HIV population to AIDS (acquired immunodeficiency syndrome) in children manifestation And Estimation Rate Death In Children.An adaptive neural network position control of a class of multi-input multi-output (MIMO) nonlinear systems called "system of ball on a sphere" in 
presence of random disturbance is presentedin [4]. By considering an HIV model, based on optimal control, the best treatment strategy is identified by using chemotherapies with minimum side effects in order to maximize the healthy cells. Also, a new approach is introduced which transform the constraints of problem to the integral constraints [5]. By an approximation, a finite dimensional linear programming problem is obtained which give an approximate solution for original problem. The arc length control via SCARA robot for welding a circular path is studiedin [6].In [7], by using fuzzy sliding control, the inverted pendulum angle is controlledby nonlinear equations. $E$. Sareban et al. [8] focuses on evolving of two types fuzzy and classical PID liquid level controller and examining whether they are better able to handle modeling uncertainties.An adaptive fuzzy controller is designed by Chiou et al. [9]neural networks and adaptive feedback linearization method are common controller which is used to robot control applications $[10,13]$. Anadaptive neuro-fuzzy controller is presented by Tian et al. [11] Moreover, other controllers have been proposed to control the robot, for example, the robust controller reported by Spong [12]. The regulation and tracking purpose for Overhead Gantry Crane Systemare considered in [14].

This paper is included to several sections: modeling and governing equations aredescribed in section 2. Section 3 is presented to explain controller and its specifications. Stochastic feedback linearization controller is designed to control the robot manipulator. The parameters are estimated byKalman filter. Also, the deference of original signals and noise signal are specified by Kalman filter. In fourth section, the simulation results are demonstrated, the figures are pictured and the yielded tracking simulation are illustrated.

\section{Robot modeling}

In this section, the dynamic of RRRRRR robot type with 6-DOF is obtained and is investigated.The schematic of the robot and its parameters are illustrated in figure1.

This robot is composed of seven links. These links are considered as solid and rigid, and named from link 0 to link 6.The significant coordinate is considered as fixed on the link, for each link, and is called as frame which is shown in figure 1 . The frame 0 is fixed on the space and is considered as universal reference frame. A moment is considered as input to controlling robot for each link and also is shown in figure 1.
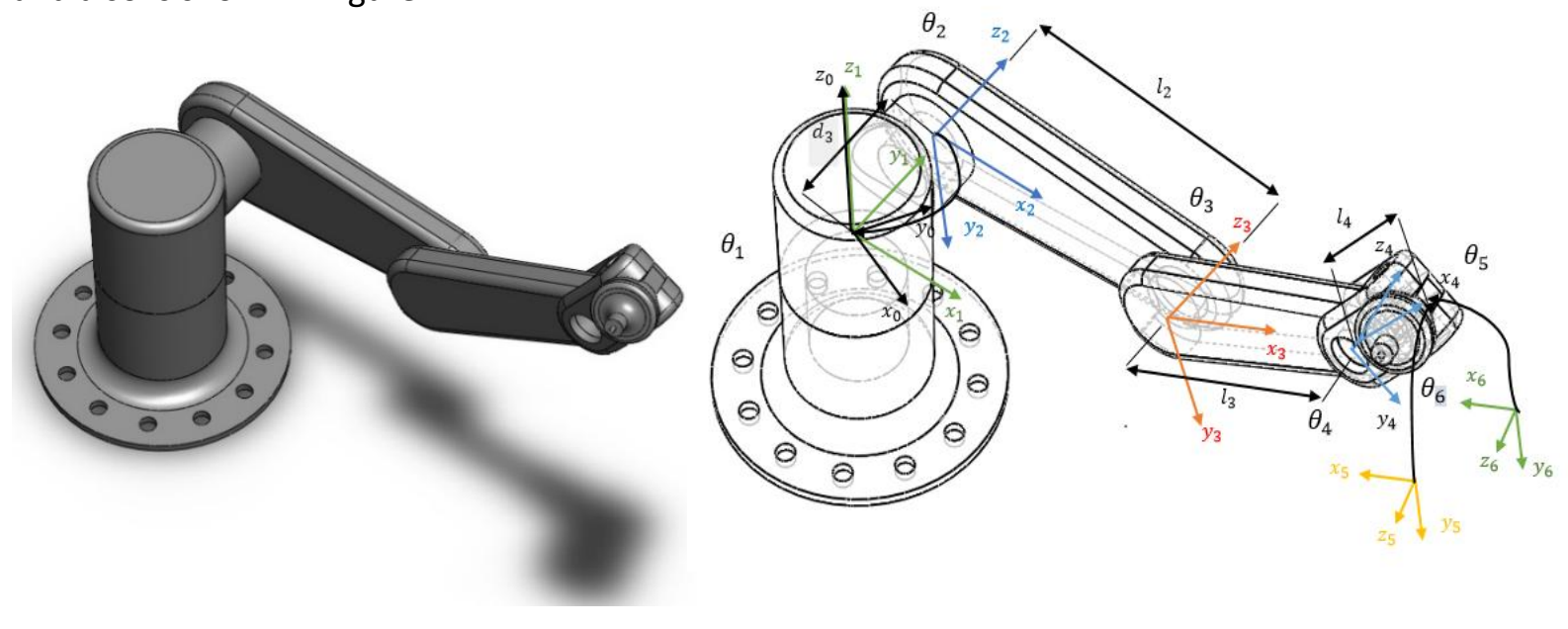

Figure 1. Schematic of PUMA 560 robot

The Euler-Lagrangian equation is used for deriving the dynamic equations. For this purpose, the kinematic and potential energy of the robot are obtained as: 


$$
\begin{aligned}
& \mathrm{K}=\Sigma \frac{1}{2} \mathrm{~m}_{\mathrm{i}}\left(\mathrm{i}_{\mathrm{V}_{\mathrm{G}}}\right)^{\mathrm{T}}\left(\mathrm{i}_{\mathrm{V}_{\mathrm{G}}}\right)+\frac{1}{2}\left(\mathrm{i}_{\mathrm{W}_{\mathrm{i}}}\right)\left(\mathrm{c}_{\mathrm{i}} \mathrm{I}_{\mathrm{i}}\right)\left(\mathrm{i}_{\mathrm{W}_{\mathrm{i}}}\right) \\
& \mathrm{U}=\Sigma \mathrm{m}_{\mathrm{i}} \mathrm{gZ}_{\mathrm{m}_{\mathrm{i}}}
\end{aligned}
$$

Where, $m_{i}$ is mass of $i_{t h}$ link, $\left(c_{i} I_{i}\right)$ is $3 \times 3$ inertia matrix around center of mass and in link frame direction of $i_{t h}$ link, $\left(i_{w_{i}}\right)$ is the $3 \times 1$ vector of absolute rotational velocity of $i_{t h}$ linkin its frame, $\left(i_{v_{G}}\right)$ is the $3 \times 3$ vector ofabsolutelinear center of mass velocity of $i_{t h}$ link, $Z_{m_{i}}$ is the center of mass height of $i_{\text {th }}$ link, and $g$ is gravity acceleration.

$\left(Z_{m_{i}}\right),\left(i_{w_{i}}\right)$ and $\left(i_{v_{G}}\right)$ is obtained from relations in robotic,as:

$$
Z_{m_{i}}=Z_{m_{i}}=\left({ }_{i}^{0} T\left(i_{P_{m_{i}}}\right)\right)^{T}\left[\begin{array}{l}
0 \\
0 \\
1
\end{array}\right]
$$

Where, ${ }_{i}^{0} T$ defined as:

$$
\begin{gathered}
{ }_{i}^{0} T={ }_{1}^{0} T \times{ }_{2}^{1} T \times \cdots \times{ }_{i}^{i-1} T \\
i+1_{W_{i+1}}={ }_{i}^{i+1} R i_{W_{i}}+\dot{\theta}_{i+1} i+{ }^{1} \hat{Z}_{i+1}
\end{gathered}
$$

And also,

$$
\begin{gathered}
\dot{\theta}_{i+1} i+1_{\hat{Z}_{i+1}}=\left[\begin{array}{c}
0 \\
0 \\
\dot{\theta}_{i+1}
\end{array}\right] \\
i_{v_{G}}={ }_{i}^{i+1} R\left(i_{v_{i}}+i_{w_{i}} \times i_{P_{m_{i}}}\right)
\end{gathered}
$$

Where, $i_{v_{i}}$ defined as:

$$
\begin{gathered}
i+1_{v_{i+1}}={ }_{i}^{i+1} R\left(i_{v_{i}}+i_{W_{i}} \times i_{P_{i+1}}\right) \\
1_{W_{1}}=\left[\begin{array}{l}
0 \\
0 \\
\dot{\theta}_{1}
\end{array}\right] \\
1_{v_{1}}=\left[\begin{array}{l}
0 \\
0 \\
0
\end{array}\right]
\end{gathered}
$$

In this equations, $i_{P_{i+1}}$ is the $3 \times 1$ vector in center of mass coordinate of $i_{t h}$ link in its frame, ${ }_{i}^{i-1} T$ is $3 \times 3$ transport matrix form $i_{t h}$ frame to $(i-1)_{t h}$ frame and ${ }_{i}^{i-1} R$ is $3 \times 3$ rotation matrix from $(i-1)_{t h}$ frame to $i_{t h}$ frame.${ }_{i}^{i-1} T$, and ${ }_{i}^{i-1} R$ is defined, as:

$$
\begin{aligned}
{ }_{\mathrm{i}} \mathrm{T} & =\left[\begin{array}{cccc}
\cos \theta_{\mathrm{i}} & -\sin \theta_{\mathrm{i}} & 0 & \mathrm{a}_{\mathrm{i}-1} \\
\sin \theta_{\mathrm{i}} \cos \alpha_{\mathrm{i}-1} & \cos \theta_{\mathrm{i}} \cos \alpha_{\mathrm{i}-1} & -\sin \alpha_{\mathrm{i}-1} & -\sin \alpha_{\mathrm{i}-1} \mathrm{~d}_{\mathrm{i}} \\
\sin \theta_{\mathrm{i}} \sin \alpha_{\mathrm{i}-1} & \cos \theta_{\mathrm{i}} \sin \alpha_{\mathrm{i}-1} & \cos \alpha_{\mathrm{i}-1} & \cos \alpha_{\mathrm{i}-1} \mathrm{~d}_{\mathrm{i}} \\
0 & 0 & 0 & 1
\end{array}\right] \\
{ }_{\mathrm{i}-1} \mathrm{R} & =\left[\begin{array}{ccc}
\cos \theta_{\mathrm{i}} & -\sin \theta_{\mathrm{i}} & 0 \\
\sin \theta_{\mathrm{i}} \cos \alpha_{\mathrm{i}-1} & \cos \theta_{\mathrm{i}} \cos \alpha_{\mathrm{i}-1} & -\sin \alpha_{\mathrm{i}-1} \\
\sin \theta_{\mathrm{i}} \sin \alpha_{\mathrm{i}-1} & \cos \theta_{\mathrm{i}} \sin \alpha_{\mathrm{i}-1} & \cos \alpha_{\mathrm{i}-1}
\end{array}\right]^{\mathrm{T}}
\end{aligned}
$$

Where, $\theta_{i}, a_{i}, \alpha_{i}$, and $d_{i}$ arelisted in Denavit-Hartenberg table from robot properties in table 1 . 


\section{TABLE 1. LINK PARAMETERS FOR PUMA -560}

\begin{tabular}{c|cccc}
\hline $\mathbf{i}$ & $\mathrm{a}_{\mathrm{i}-1}$ & $\alpha_{\mathrm{i}-1}$ & $\mathrm{~d}_{\mathrm{i}}$ & $\theta_{\mathrm{i}}$ \\
$\mathbf{1}$ & 0 & 0 & 0 & $\theta_{1}$ \\
$\mathbf{2}$ & 0 & -90 & 0 & $\theta_{2}$ \\
$\mathbf{3}$ & $\mathrm{L}_{2}$ & 0 & $\mathrm{~d}_{3}$ & $\theta_{3}$ \\
$\mathbf{5}$ & $\mathrm{L}_{3}$ & 0 & 0 & $\theta_{4}$ \\
$\mathbf{6}$ & $\mathrm{L}_{4}$ & 90 & 0 & $\theta_{5}$ \\
& 0 & -90 & 0 & $\theta_{6}$ \\
\hline
\end{tabular}

Finally, the dynamic equation is obtained from Euler-Lagrangian equation, as:

$$
\frac{\mathrm{d}}{\mathrm{dt}}\left(\frac{\partial \mathrm{L}}{\partial \mathrm{q}}\right)+\frac{\partial \mathrm{L}}{\partial \mathrm{q}_{1}}=\mathrm{F}
$$

Where, $L$ is the Lagrangian function, $q$ is the states vector and $F$ is system inputs which are defined as:

$$
\begin{gathered}
\mathrm{L}=\mathrm{K}-\mathrm{U} \\
\mathrm{q}=\left[\begin{array}{llllll}
\theta_{1} & \theta_{2} & \theta_{3} & \theta_{4} & \theta_{5} & \theta_{6}
\end{array}\right]^{\mathrm{T}} \\
\mathrm{F}=\left[\begin{array}{lllllll}
\mathrm{M}_{1} & \mathrm{M}_{2} & \mathrm{M}_{3} & \mathrm{M}_{4} & \mathrm{M}_{5} & \mathrm{M}_{6}
\end{array}\right]^{\mathrm{T}}
\end{gathered}
$$

The dynamic model of the PUMA 560 can be expressed in the form of matrix from 13, as:

$$
M(q) \ddot{q}+C(q, \dot{q})+G(q)=F
$$

Where, the matrices $M(q) \in \mathcal{R}^{6 \times 6}, C(q, \dot{q}) \in \mathcal{R}^{6 \times 1}$ and $G(q) \in \mathcal{R}^{6 \times 1}$ represent the inertia, centripetalCoriolis terms and gravity, respectively.

So the system dynamic equation of PUMA 560 in the state space is formed as below:

$$
\begin{gathered}
\ddot{\mathrm{q}}=\mathrm{M}^{-1}(\mathrm{~F}-\mathrm{C}(\mathrm{q}, \dot{\mathrm{q}})-\mathrm{G}(\mathrm{q})) \\
x_{1}=q_{1}, x_{2}=\dot{q}_{1}, x_{3}=q_{2}, x_{4}=\dot{q}_{2}, x_{5}=q_{3}, x_{6}=\dot{q}_{3}, x_{7}=q_{4}, x_{8}=\dot{q}_{4}, x_{9}=q_{5}, x_{10} \\
=\dot{q}_{5}, x_{11}=q_{6}, x_{12}=\dot{q}_{6}
\end{gathered}
$$

And derivation of states is

$$
\begin{gathered}
\dot{\mathrm{x}}_{1}=\mathrm{x}_{2}, \dot{\mathrm{x}}_{2}=\ddot{\mathrm{q}}_{1}, \dot{\mathrm{x}}_{3}=\mathrm{x}_{4}, \dot{\mathrm{x}}_{4}=\ddot{\mathrm{q}}_{2}, \dot{\mathrm{x}}_{5}=\mathrm{x}_{6}, \dot{\mathrm{x}}_{6}=\ddot{\mathrm{q}}_{3}, \dot{\mathrm{x}}_{7}=\mathrm{x}_{8}, \dot{\mathrm{x}}_{8}=\ddot{\mathrm{q}}_{4}, \dot{\mathrm{x}}_{9}=\mathrm{x}_{10}, \dot{\mathrm{x}}_{10} \\
=\ddot{\mathrm{q}}_{5}, \dot{\mathrm{x}}_{11}=\mathrm{x}_{12}, \dot{\mathrm{x}}_{12}=\ddot{\mathrm{q}}_{6}
\end{gathered}
$$

\section{Control design}

In this section,the process of controller design is presented. In this study,stochastic feedback linearization with Kalman filter is used to control the PUMA 560 robot system. Feedback 
linearization method is useful procedure to nonlinear system and it is used by enormous researchers [14]. The schematic of the controller with system planning is pictured in figure 2.

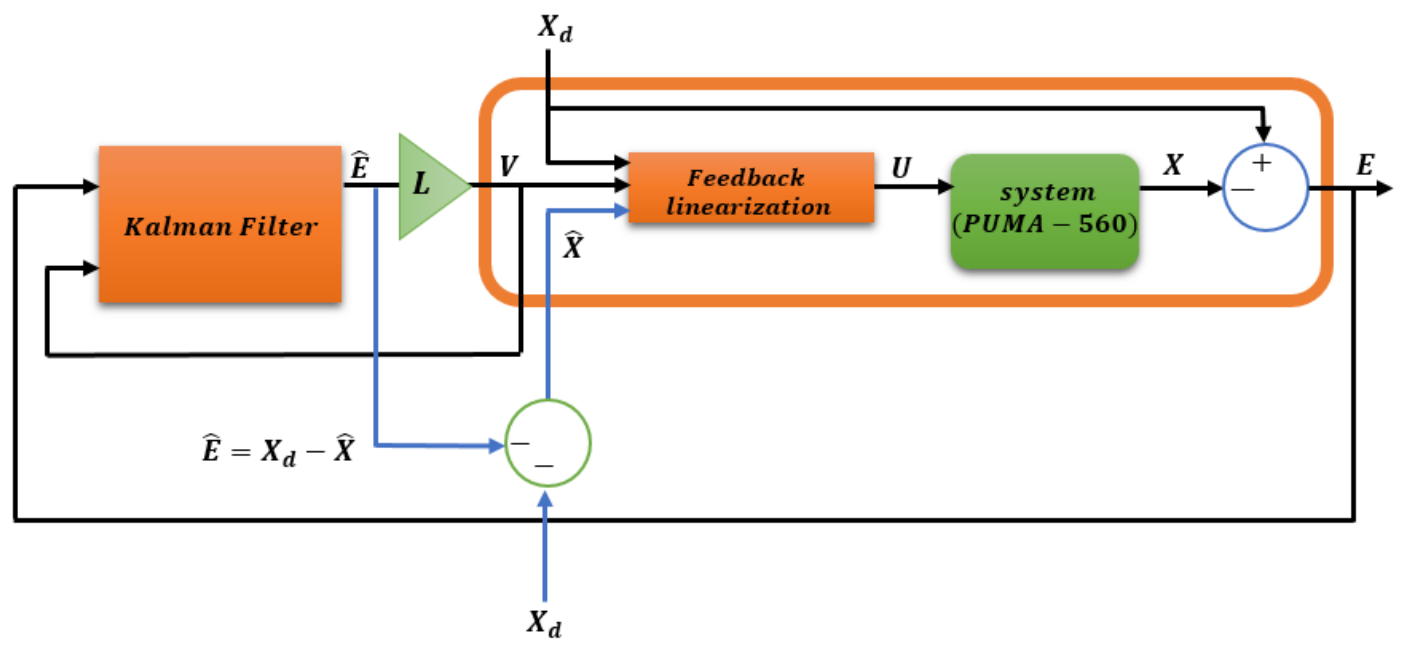

Figure 2. Schematic of designed PUMA 560 controller

\subsection{Feedback linearization}

The equivalent stable linear system is substituted to the original nonlinear system due tofeedback linearization technique. The process of deriving control rule from the nonlinear system is shown in below. By considering the stable linear system:

$$
\dot{E}=A E+B V
$$

Where, matrix $A$ and $B$ be are defied as:

$$
A=\left[\begin{array}{cccccccccccc}
0 & 1 & 0 & 0 & 0 & 0 & 0 & 0 & 0 & 0 & 0 & 0 \\
-1 & -1 & 0 & 0 & 0 & 0 & 0 & 0 & 0 & 0 & 0 & 0 \\
0 & 0 & 0 & 1 & 0 & 0 & 0 & 0 & 0 & 0 & 0 & 0 \\
0 & 0 & -1 & -1 & 0 & 0 & 0 & 0 & 0 & 0 & 0 & 0 \\
0 & 0 & 0 & 0 & 0 & 1 & 0 & 0 & 0 & 0 & 0 & 0 \\
0 & 0 & 0 & 0 & -1 & -1 & 0 & 0 & 0 & 0 & 0 & 0 \\
0 & 0 & 0 & 0 & 0 & 0 & 0 & 1 & 0 & 0 & 0 & 0 \\
0 & 0 & 0 & 0 & 0 & 0 & -1 & -1 & 0 & 0 & 0 & 0 \\
0 & 0 & 0 & 0 & 0 & 0 & 0 & 0 & 0 & 1 & 0 & 0 \\
0 & 0 & 0 & 0 & 0 & 0 & 0 & 0 & -1 & -1 & 0 & 0 \\
0 & 0 & 0 & 0 & 0 & 0 & 0 & 0 & 0 & 0 & 0 & 1 \\
0 & 0 & 0 & 0 & 0 & 0 & 0 & 0 & 0 & 0 & -1 & -1
\end{array}\right]
$$




$$
B=\left[\begin{array}{llllll}
0 & 0 & 0 & 0 & 0 & 0 \\
1 & 0 & 0 & 0 & 0 & 0 \\
0 & 0 & 0 & 0 & 0 & 0 \\
0 & 1 & 0 & 0 & 0 & 0 \\
0 & 0 & 0 & 0 & 0 & 0 \\
0 & 0 & 1 & 0 & 0 & 0 \\
0 & 0 & 0 & 0 & 0 & 0 \\
0 & 0 & 0 & 1 & 0 & 0 \\
0 & 0 & 0 & 0 & 0 & 0 \\
0 & 0 & 0 & 0 & 1 & 0 \\
0 & 0 & 0 & 0 & 0 & 0 \\
0 & 0 & 0 & 0 & 0 & 1
\end{array}\right]
$$

Where, $E$ is the state variable vector of the linear system and $V$ is $6 \times 1$ vector of system input. Also, the nonlinear system is obtained as:

$$
\dot{X}=H(X)+G(X) U
$$

Where, $X$ is the state variable of the nonlinear system, $H$ and $G$ are derived from equation 20. By considering $E$ as error we have:

$$
\begin{gathered}
E=X_{d}-X \\
\dot{E}=\dot{X}_{d}-\dot{X}=X_{d}-(H(X)+G(X) U)
\end{gathered}
$$

Then

$$
\dot{X}_{d}-(H(X)+G(X) U)=A E+B V
$$

So, the controller law " $\mathrm{U}$ " is derived by solving the even rows of above equation for nonlinear system.

\subsection{LQEestimator (Kalman filter)}

In control theory, the Linear-Quadratic-Estimator (LQE) control problem is one of the most fundamental optimal control problems. It concerns uncertain linear systems disturbed by additive white Gaussian noise, having incomplete state information and undergoing control subject to quadratic costs. Moreover, the solution is unique and constitutes a linear dynamic feedback control law that is easily computed and can be implemented. Finally, the LQE controller is also foundation to the optimal control of perturbed nonlinear systems.

The linear system is considered as:

$$
\begin{gathered}
\dot{E}=A E+B V+n \\
Y=C E+w
\end{gathered}
$$

In this problem $C$ is $12 \times 12$ identity matrix.

Where the vector $\mathrm{E}$ is state variable, the vector $V$ is control input, the vector $Y$ is measurement output, the vector $n$ is additive white Gaussian system noise and $w$ is additive white Gaussian measurement noise.

The LQE controller is specified by the following equations: 


$$
\begin{gathered}
\hat{\dot{E}}=A E+B V+K(Y-C \hat{E}) \\
V=-L \hat{X} \\
\hat{E}_{0}=\operatorname{Expected} \operatorname{value}\left(E_{0}\right)
\end{gathered}
$$

Where the matrix $\mathrm{K}$ is Kalman gain that is obtained from Kalman filter equation.

$$
K=P C^{T} W^{-1}
$$

Where,

$$
\begin{gathered}
\dot{P}=A \cdot P+P \cdot A^{T}-P \cdot C^{T} W^{-1} C \cdot P+N \\
P_{0}=\text { expected value }\left(E_{0} \cdot E_{0}^{T}\right)
\end{gathered}
$$

Where, $W$ is the covariance of $w$ and $N$ is the covariance of nin the above differential equations.

\section{Simulation results}

$\theta_{1}, \theta_{2}, \theta_{3}, \theta_{4}, \theta_{5}, \theta_{6}$ Arethe main parameters ofPUMA 560 robot regulating and tracking purpose,but because of the figures for all of the manipulators in this robot is large, only first three manipulator control figures of robot are presented. $\theta_{1}, \theta_{2}$ and $\theta_{3}$ are considered as main parameters to regulate and track the system angle by controller.The desired values for regulation purpose are listed in table 2. Figures 3 to 8 are presented to show resultant measured and estimated of parameters both without and in presence of disturbance.

\section{TABLE 2. DESIRED VALUES FOR REGULATION PURPOSE}

\begin{tabular}{c|c}
$\boldsymbol{\theta}_{\mathbf{1}_{\boldsymbol{d}}}$ & $0.5 \mathrm{rad}$ \\
$\boldsymbol{\theta}_{\mathbf{2}_{\boldsymbol{d}}}$ & $0.5 \mathrm{rad}$ \\
$\boldsymbol{\theta}_{\mathbf{3}_{\boldsymbol{d}}}$ & $0.5 \mathrm{rad}$
\end{tabular}




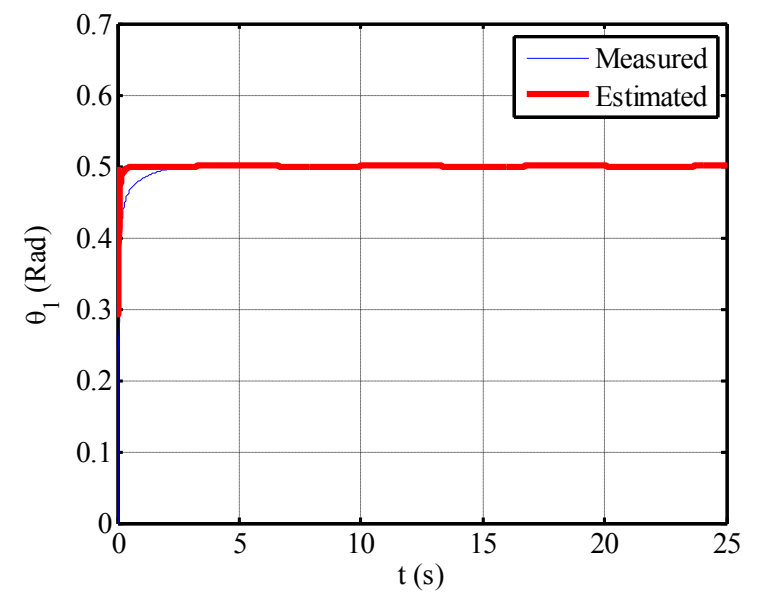

Figure 3.Measured and Estimated $\theta_{1}$ without disturbance

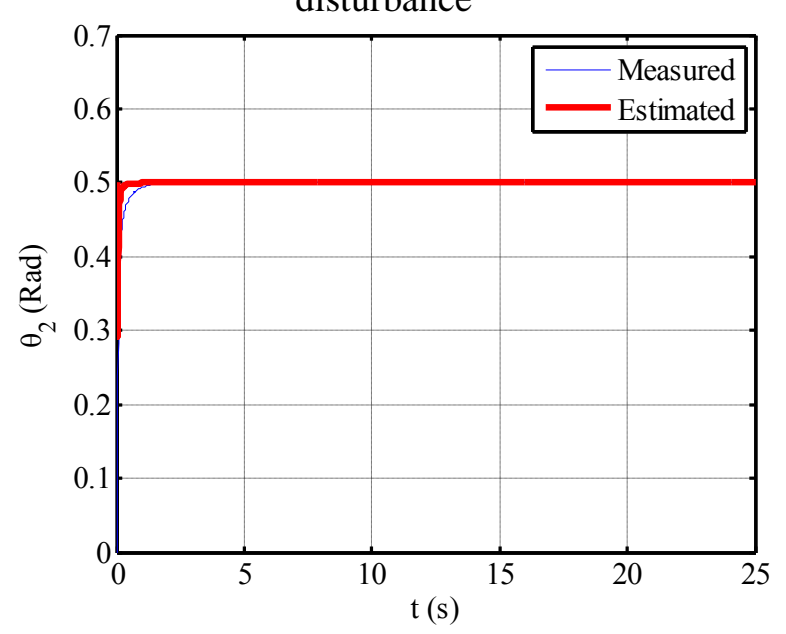

Figure 5.Measured and Estimated $\theta_{2}$ without disturbance

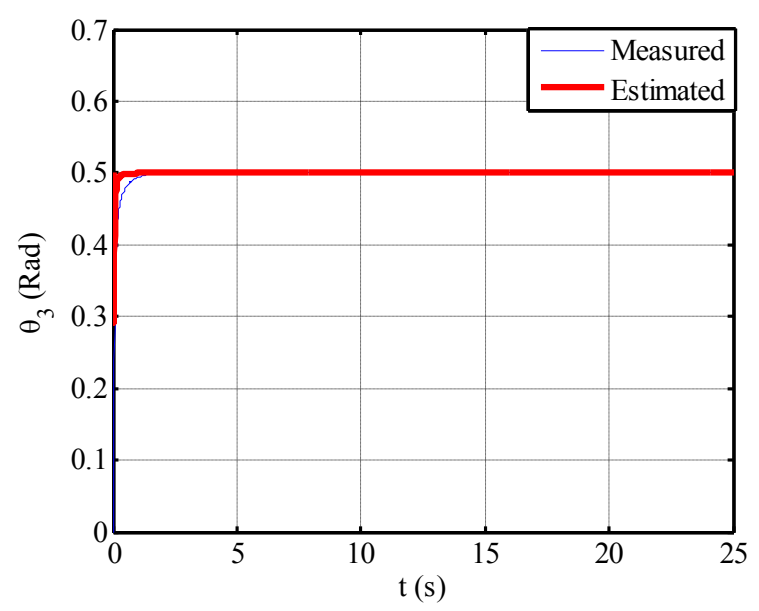

Figure 7.Measured and Estimated $\theta_{3}$ without disturbance

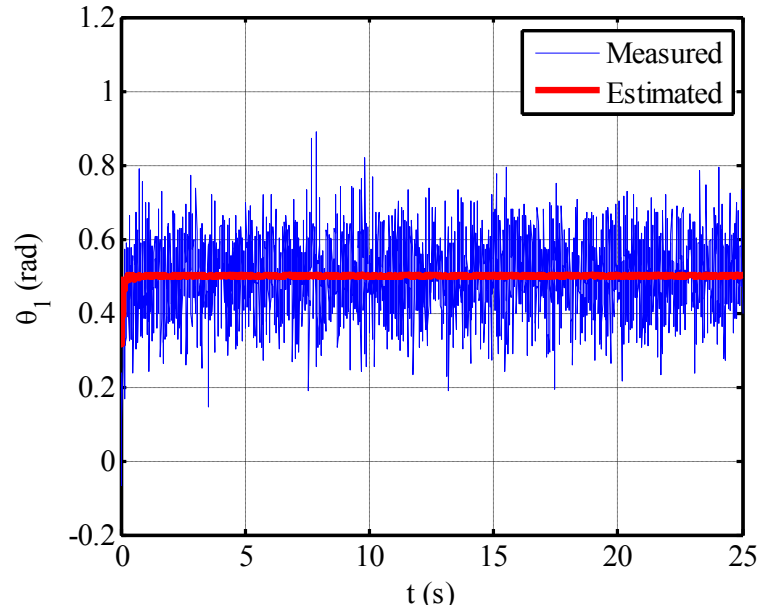

Figure 4.Measured and Estimated $\theta_{1}$ in presence of disturbance

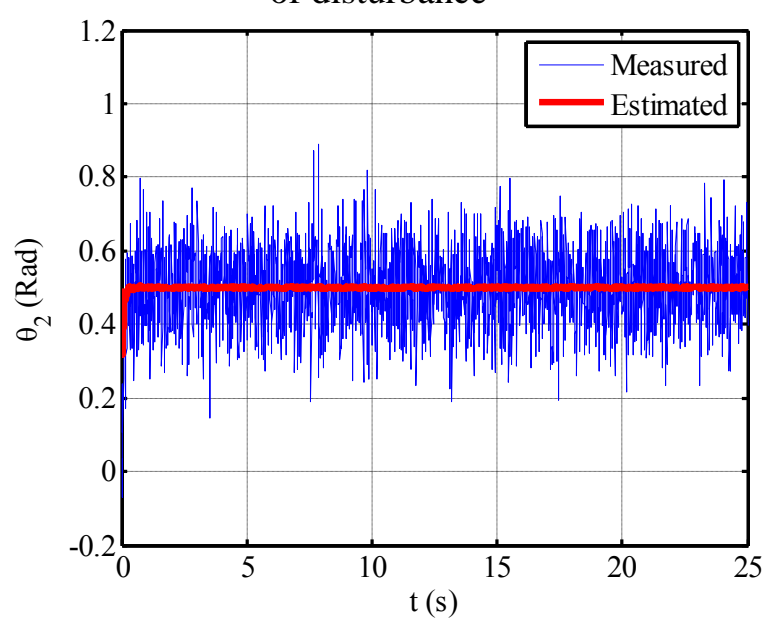

Figure 6.Measured and Estimated $\theta_{2}$ in presence of disturbance

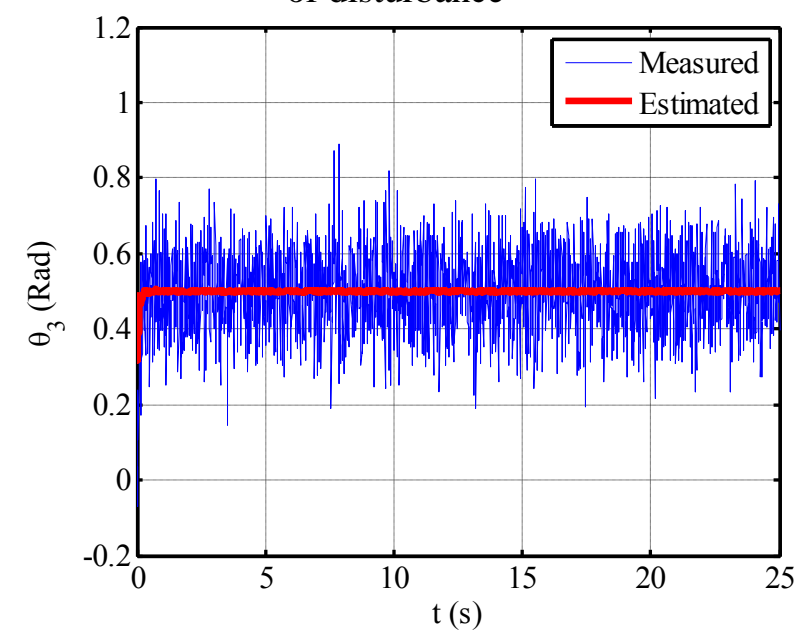

Figure 8.Measured and Estimated $\theta_{3}$ in presence of disturbance

Figures 9 to 14 are presented for regulation purpose of parameters both without and in presence of disturbance. 


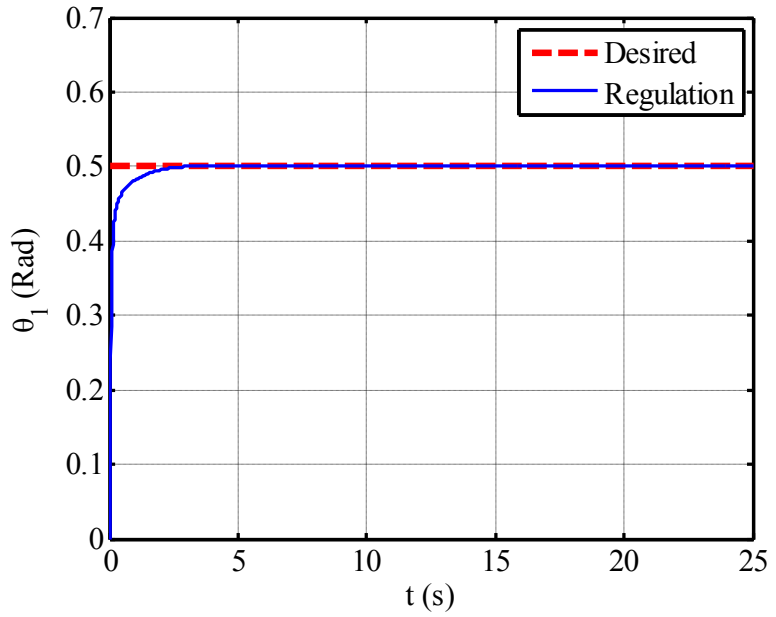

Figure 9.Regulation $\theta_{1}$ without disturbance

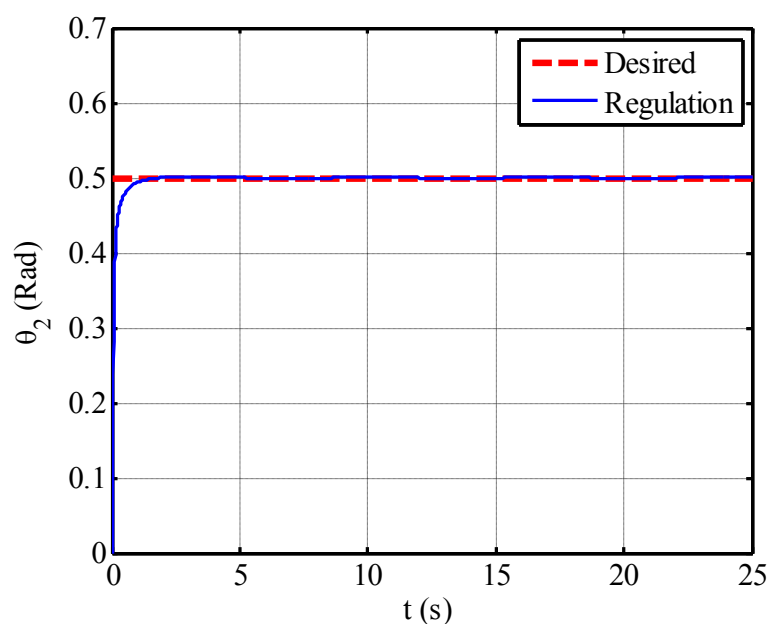

Figure 11.Regulation $\theta_{2}$ without disturbance

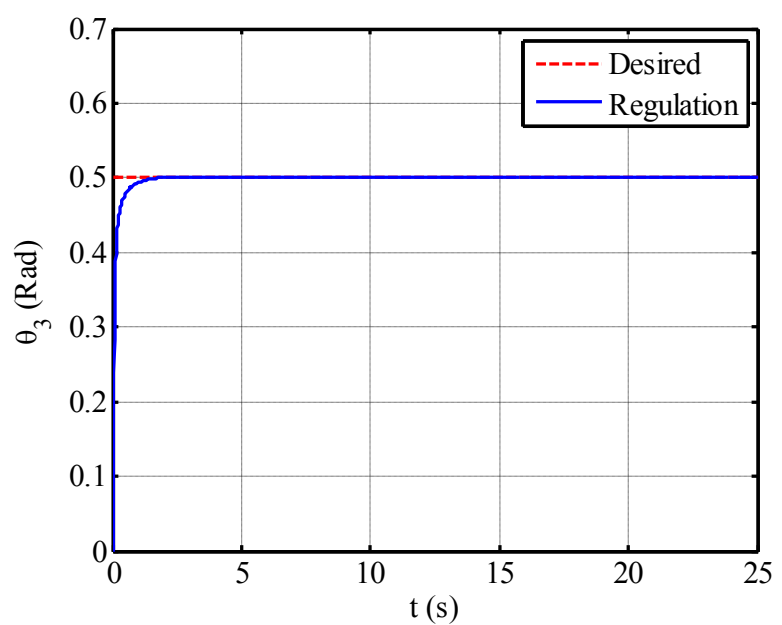

Figure 13. $\operatorname{Regulation} \theta_{3}$ without disturbance

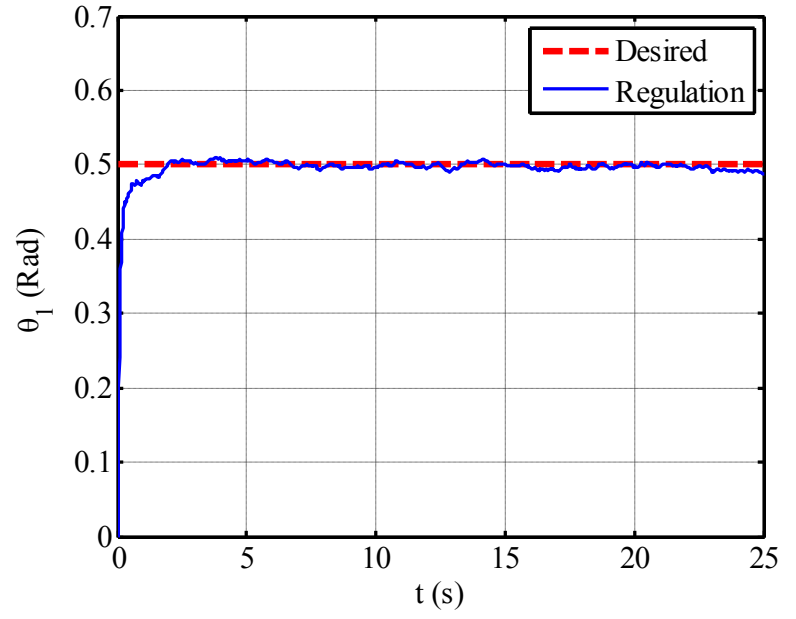

Figure 10.Regulation $\theta_{1}$ in presence of disturbance

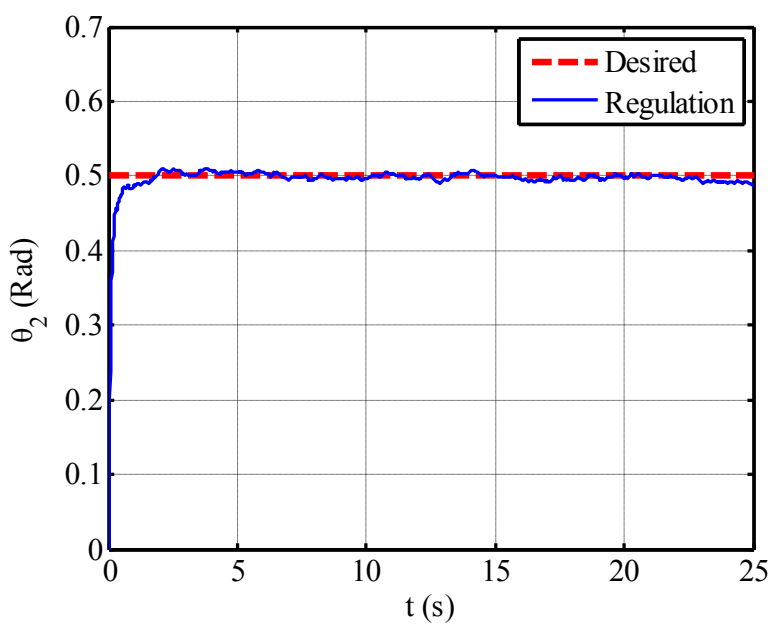

Figure 12. Regulation $\theta_{2}$ in presence of disturbance

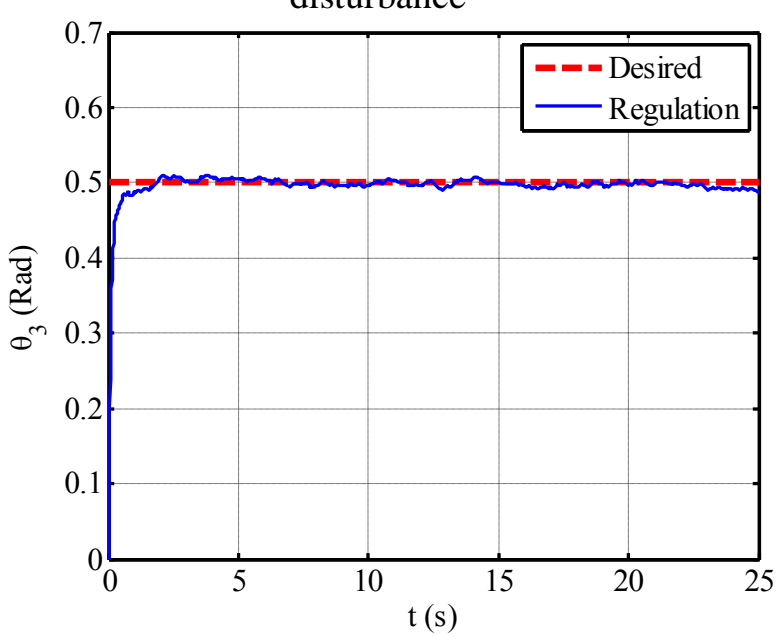

Figure 14.Regulation $\theta_{3}$ in presence of disturbance

Figures 15 to 20 are presented for tracking purpose for measured and estimated parameters. 


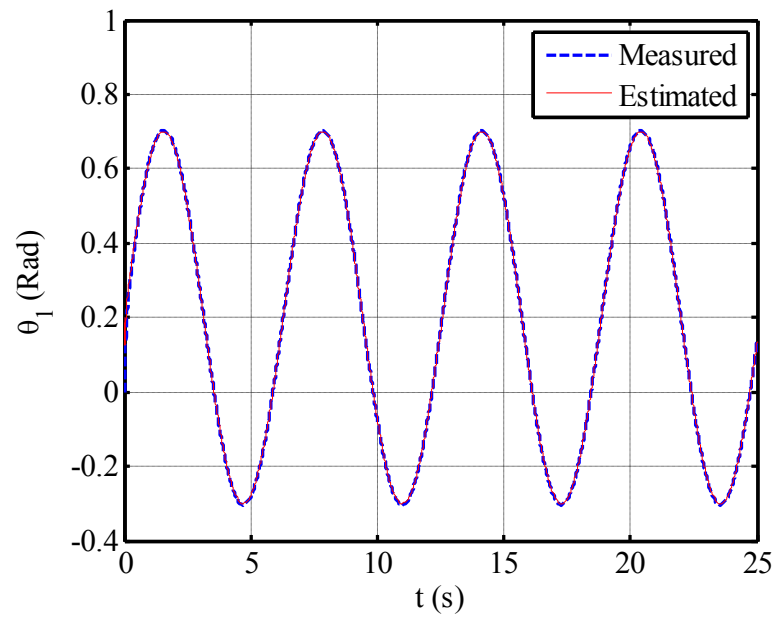

Figure 15.Measured and Estimated $\theta_{1}$ without disturbance

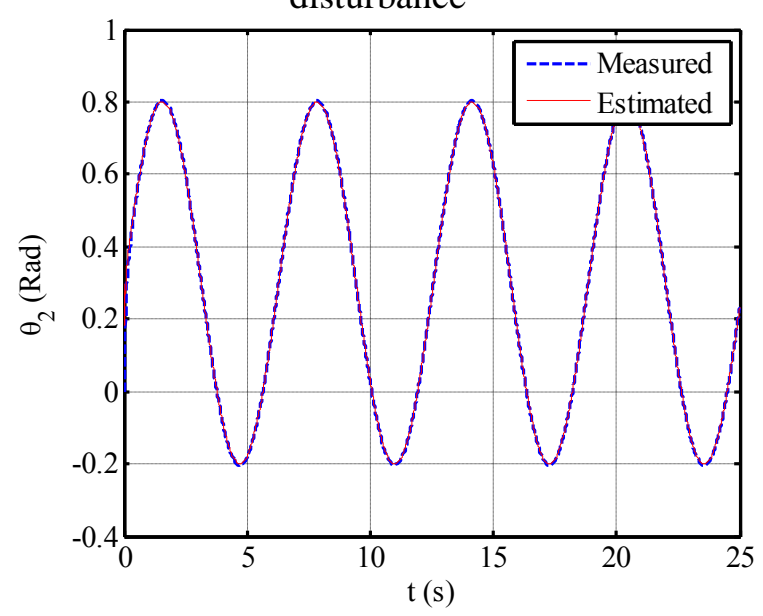

Figure 17.Measured and Estimated $\theta_{2}$ without disturbance

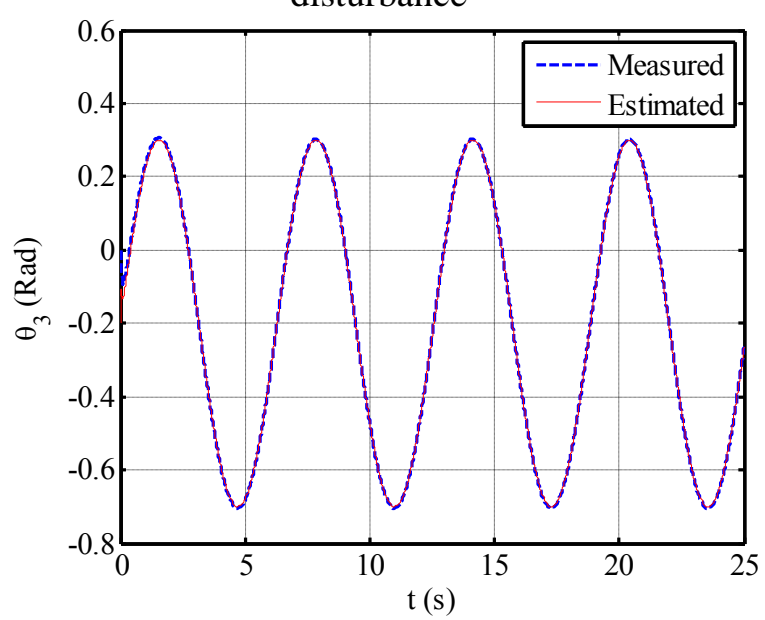

Figure 19.Measured and Estimated $\theta_{3}$ without disturbance

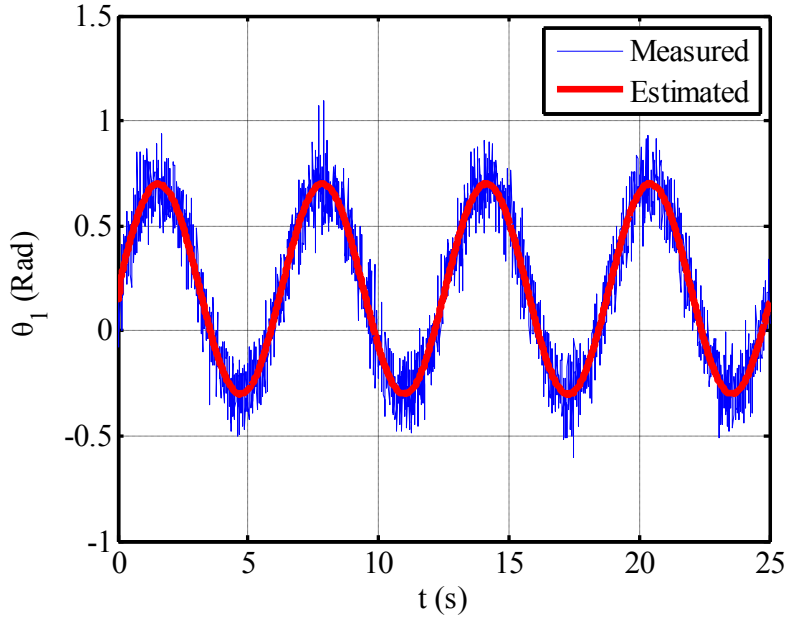

Figure 16. Measured and Estimated $\theta_{1}$ in presence of disturbance

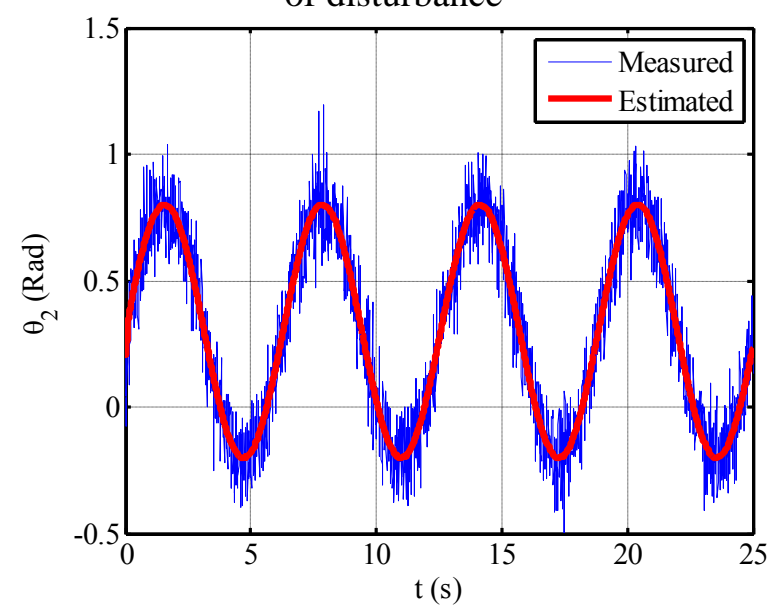

Figure 18. Measured and Estimated $\theta_{2}$ in presence of disturbance

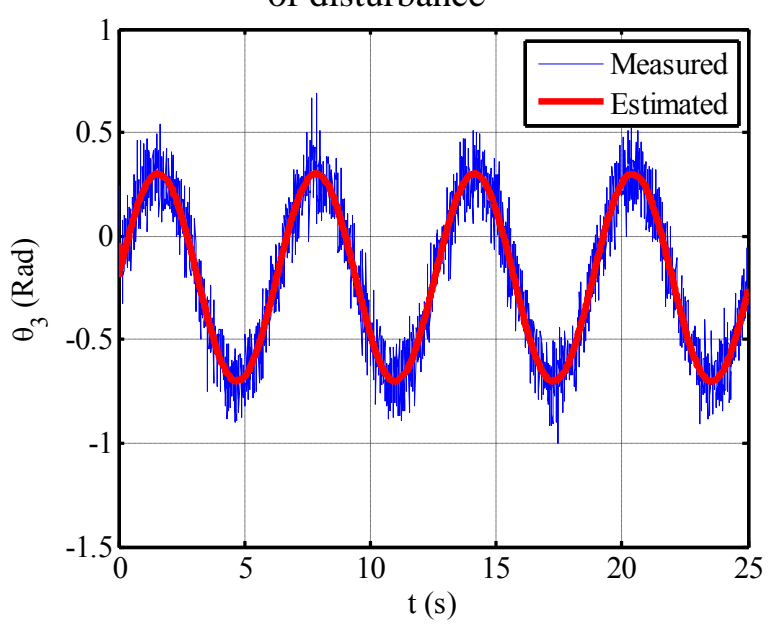

Figure 20.Measured and Estimated $\theta_{3}$ in presence of disturbance

Figures 21 to 26 are presented for tracking purpose of parameters both without and in presence of disturbance. 


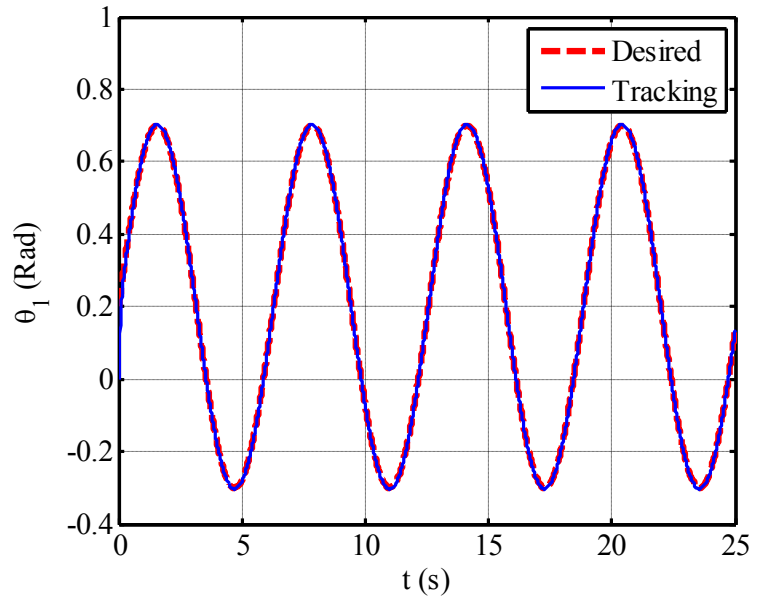

Figure 21. Tracking $\theta_{1}$ without disturbance

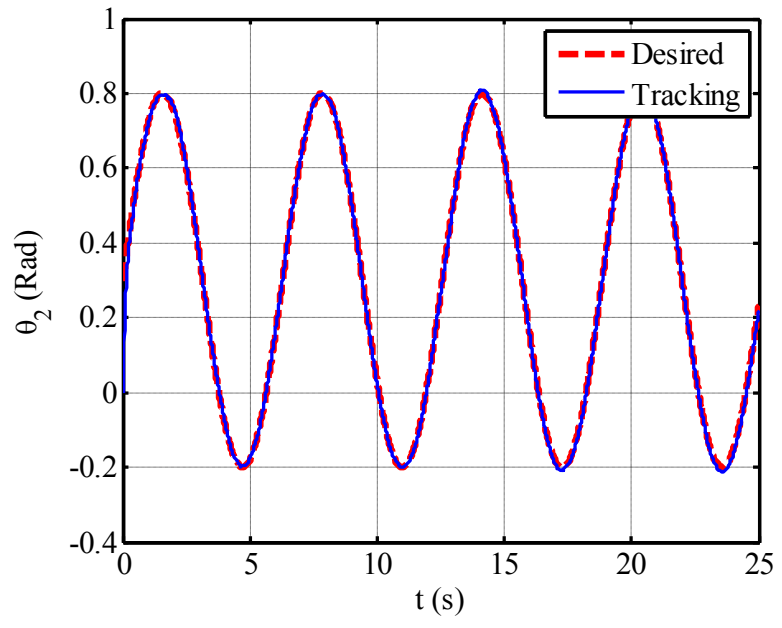

Figure 23.Tracking $\theta_{2}$ without disturbance

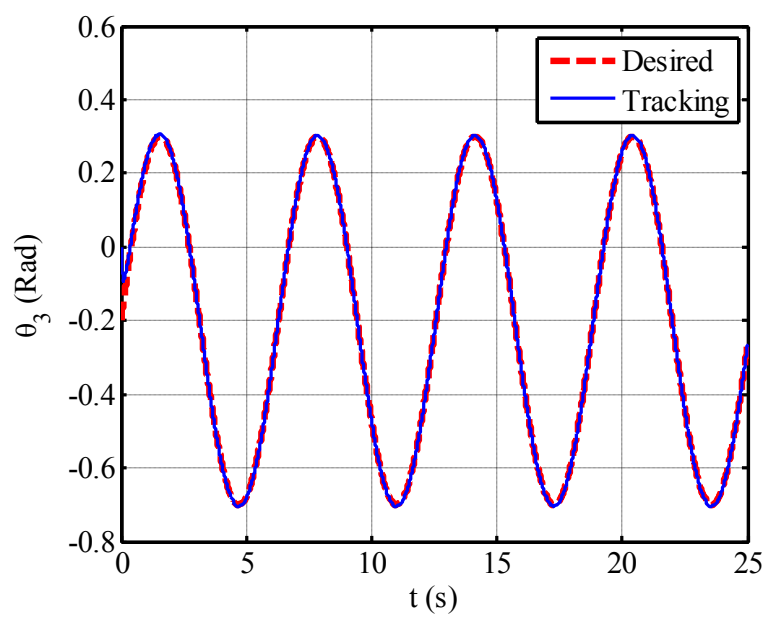

Figure 25.Tracking $\theta_{3}$ without disturbance

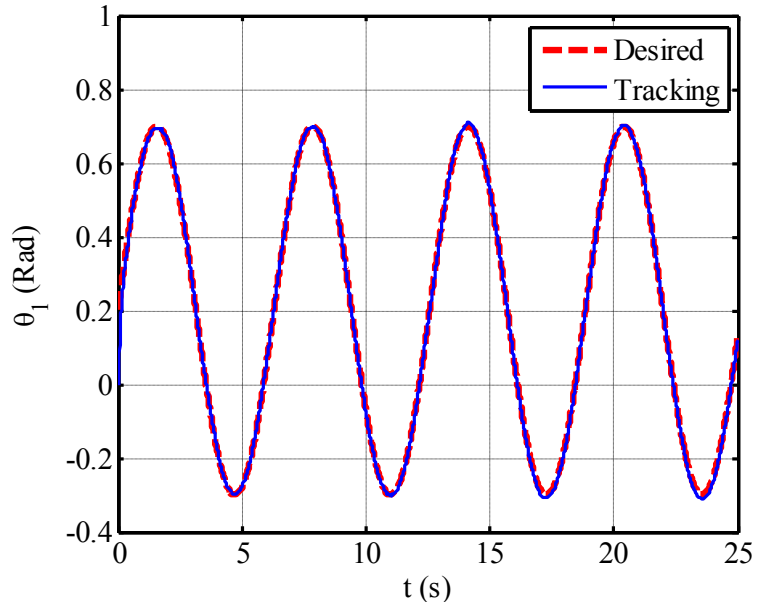

Figure 22.Tracking $\theta_{1}$ in presence of disturbance

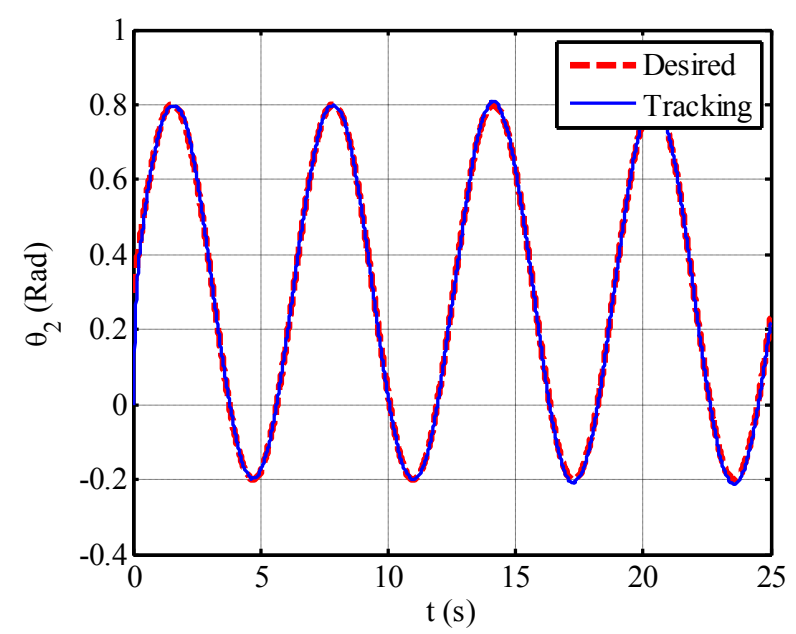

Figure 24.Tracking $\theta_{2}$ in presence of disturbance

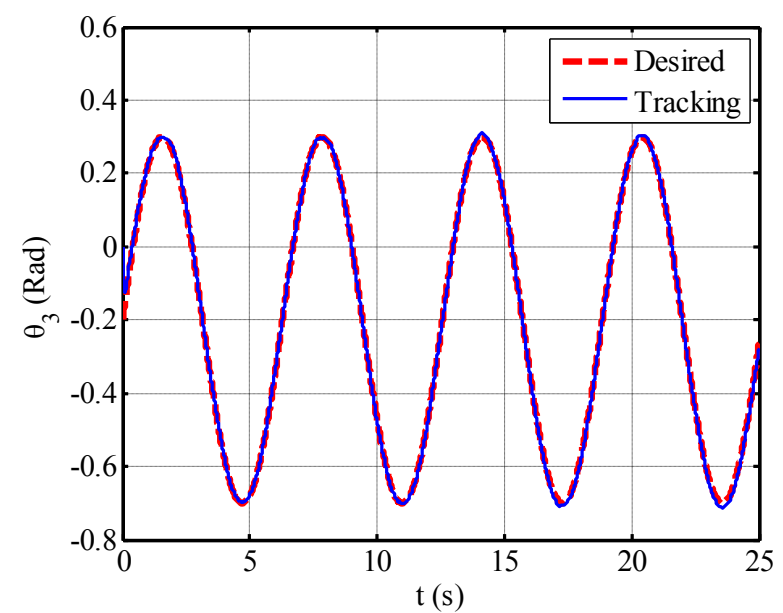

Figure 26.Tracking $\theta_{3}$ in presence of disturbance 


\section{Conclusion}

In this study, the PUMA 560 robot with three degree of freedom is modeled. Then, it is controlled by stochastic feedback linearization LQE controller. This robot is RRRRRR robot type. The dynamic model is derived by Euler-Lagrange method. The performance of the controllers is examined for regulating and tracking purpose of manipulators by desired inputs. The controller is successfully regulated in presence of the disturbances which is confirmed by figures and curves.

\section{References}

[1] J. Mozaryn, J.E., Kurek, "Design of decoupled sliding mode control for the PUMA 560 robot manipulator," Robot Motion and Control, 2002. RoMoCo '02. Proceedings of the Third International Workshop on, vol., no., pp.45,50, 9-11 Nov. 2002

[2] A.K. Bejczy, T.-J. Tarn, X. Yun, S. Han, "Nonlinear feedback control of PUMA 560 robot arm by computer," Decision and Control, 1985 24th IEEE Conference on, vol.24, no., pp.1680,1688, Dec. 1985

[3] A. Vahidian Kamyad, M. Shokohi Nia, M.R. Shokohi Nia, "the Estimation of Transference Rate HIV Infection into AIDS and Mortality in Children by Fuzzy Control” Volume: 2 (2011) Issue: 2 Pages: 241 - 254

[4] S. A. Moezi, E. Zakeri, Y. Bazargan-Lari, M. Tavallaeinejad "Control of a Ball on Sphere System with Adaptive Neural Network Method for Regulation Purpose”Journal of Applied Sciences 14 (17), 1984-1989

[5] H. R. Erfanian and M. H. Noori Skandari, Optimal Control of an HIV Model, The Journal of Mathematics and Computer Science, Vol2_Iss2_241-254

[6] Ehsan Zakeri, Yousef Bazargan-Lari and Mohammad Eghtesad, 2012. Simultaneous Control of GMAW Process and SCARA Robot in Tracking a Circular Path via a Cascade Approach. Trends in Applied Sciences Research, 7: 845-858

[7] M.R. Dastranj, M. Moghaddas, K. Esmaeili Khoshmardan, A. Zare "Robust Control of Inverted Pendulum Using Fuzzy Sliding Mode Control (FSMC)’The Journal of Mathematics and Computer Science, Vol .2 No.4 (2011) 659-666

[8] E. Sareban, A. Aminian, S. K Mousavi Mashhadi, "Synthesis Water Level Control by Fuzzy Logic", The Journal of Mathematics and Computer Science,9 (2014) $300-313$

[9] K.Ch. Chiou, S.J. Huang, “An adaptive fuzzy controller for robot manipulators”, Mechatronics, Volume 15, Issue 2, March 2005, Pages 151-177

[10] Y. Tang, F. Sun, and Z. Sun, "Neural Network Control of Flexible-link Manipulators Using Sliding Mode," neuro computing, vol. 70, pp. 288-295, 2006

[11] L. Tian and C. Collins, "Adaptive Neuro-Fuzzy Control of a Flexible Manipulator” Mechatronics, vol. 15, pp. 13051320,2005

[12] M. W. Spong, “On the robust control of robot manipulators," IEEE Trans. On Automatic Control, vol. 37, no. 11, pp. 1782-1786, November 1992.

[13] E. Zakeri, S. A. Moezi, Y. Bazargan-Lari, "Control of a Ball on Sphere System with Adaptive Feedback Linearization method for regulation purpose”, MAJLESI JOURNAL OF MECHATRONIC SYSTEMS. 10/2013; 2(3). 
[14] Y. Bazargan-Lari, E. Zakeri, A. R. Ghahramani, K. Bazargan-Lari, S. A. Moezi, "Disturbance Rejection Control of 3-D Overhead Gantry Crane System for Regulation purpose", National Conference on Mechanical Engineering February 22-23, 2012, Islamic Azad University, Shiraz Branch, Shiraz, Iran; 02/2012. 